\title{
Volatile Organic Compounds to Identify Infectious (Bacteria/Viruses) Diseases of the Central Nervous System - A Pilot Study
}

\section{Lei Guo}

Department of Anesthesiology, the First Affiliated Hospital of Harbin Medical University, Harbin

\section{Zhongzhi Qiu}

Department of Anesthesiology, the First Affiliated Hospital of Harbin Medical University,Harbin

\section{Yue Wang}

Department of Anesthesiology, the Fifth Affiliated Hospital of Sun Yat-Sen University,Zhuhai

\section{Kaili Yu}

Department of Critical Care Medicine,Harbin Medical University Cancer Hospital,Harbin

\section{Xiaoya Zheng}

Department of Critical Care Medicine,Harbin Medical University Cancer Hospital.Harbin

\section{Yuhang Li}

Department of Critical Care Medicine,Harbin Medical University Cancer Hospital,Harbin

\section{Miao Liu}

Department of Critical Care Medicine,Harbin Medical University Cancer Hospital,Harbin

\section{Guiyue Wang}

Department of Critical Care Medicine,Harbin Medical University Cancer Hospital,Harbin

\section{Nana Guo}

Department of Critical Care Medicine,Harbin Medical University Cancer Hospital,Harbin

\section{Mengyuan Yang}

Department of Critical Care Medicine,Harbin Medical University Cancer Hospital,Harbin

\section{Enyou Li}

Department of Anesthesiology,the First Affiliated Hospital of Harbin Medical University,Harbin

Changsong Wang ( $\nabla$ changsongwangicu@163.com )

Department of Critical Care Medicine, Harbin Medical University Cancer Hospital https://orcid.org/0000-0002-0079-5259

\section{Research article}

Keywords: Volatile organic compounds; Central nervous system infectious diseases; Bacterial meningitis/encephalitis; Viral meningitis/encephalitis ; Biomarkers. 
Posted Date: June 20th, 2019

DOl: https://doi.org/10.21203/rs.2.10462/v1

License: (c) (i) This work is licensed under a Creative Commons Attribution 4.0 International License. Read Full License

Version of Record: A version of this preprint was published at European Neurology on June 28th, 2021. See the published version at https://doi.org/10.1159/000507188. 


\section{Abstract}

Background Central nervous system (CNS) infectious diseases are common diseases in emergency rooms and neurology departments. CNS pathogen identification methods are time-consuming and expensive and have low sensitivity and poor specificity. Some studies have shown that bacteria and viruses can produce specific volatile organic compounds (VOCs). The aim of this study is to find potential biomarkers by VOC analysis of cerebrospinal fluid (CSF) in patients with bacterial and viral meningitis/encephalitis (ME). Methods CSF samples from 16 patients with bacterial ME and 42 patients with viral ME were collected, and solid-phase microextraction (SPME) combined with gas chromatography-mass spectrometry (GC-MS) was used to analyze the metabolites in the CSF. Results There are two substances (Ethylene oxide and Phenol) that were found to be different between the two groups. Ethylene oxide was significantly greater in the group of bacterial ME patients than in the viral ME group of patients $(P<0.05)$. In addition, phenol was remarkably increased in the group of ME patients compared with the bacterial ME patients $(P<0.05)$. Conclusions Ethylene oxide and phenol may be potential biomarkers to distinguish bacterial ME and viral ME. VOC analysis of CSF may be used as a supporting tool for clinical diagnosis.

\section{Background}

Central nervous system (CNS) infections are a public health concern and are common diseases in emergency rooms and neurology departments [1]. CNS infectious diseases can be divided into viral infections, bacterial infections and other infections. It has been estimated that at least 275,000 deaths from bacterial meningitis have occurred among all ages in 2010 [2]. Viral meningitis is more common than bacterial meningitis [3]. Early antibiotic and antiviral therapy can improve prognosis [4-6], so it is important to identify bacterial and viral meningitis/encephalitis (ME) quickly and accurately. There are many diagnostic challenges for identifying bacterial ME versus viral ME. The clinical presentations may be varied, and symptoms such as fever, headache, neck stiffness and meningeal irritation are often similar in adult bacterial and viral ME $[3,7]$. Routine testing such as cellular and chemistry parameters in the cerebrospinal fluid (CSF) may suggest the type of infectious diseases (e.g., bacterial versus viral); however, these parameters have poor specificity [8]. For bacterial ME, culture is useful but takes 2-5 days, and if pretreatment with antibiotics or incorrect specimen handling has rendered the sample sterile, the results may be falsely negative.

In pathological conditions, the blood-brain barrier (BBB) and the blood-cerebrospinal fluid barrier (BCSFB) are damaged and the pathogen could enter into the CNS. During the course of CNS infectious disease, metabolic changes in bacterial and viral microbiological cultures can be associated with significant volatile organic compound (VOC) release by the pathogen; hence, they provide a differential fingerprint [9]. Therefore, we hypothesized that the VOCs produced by bacteria and viruses in the CNS can be used to identify bacterial and viral ME. 
There are various analytical techniques available for bacterial/viral VOC analysis, including gas chromatography (GC), mass spectrometry (MS), electronic nose (E-noses) and ion mobility spectrometry (IMS) [10]. Among them, gas chromatography-mass spectrometry (GC-MS) is a preferred method for the identification of pathogen markers with an appropriate sensitivity [11]. As a developing novel tool for noninvasive detection of various disease states in recent years, VOC analysis has been applied in different body fluids, such as blood, urine, and saliva [12-15], and it also can be applied to identify pathogens such as Escherichia coli, Staphylococcus aureus, Mycobacterium tuberculosis, Haemophilus influenzae, Streptococcus pneumoniae, rhinoviruses, influenza $A$ virus and others $[9,10,16]$. Although there are a large number of clinical studies on the diagnosis of CNS infection such as cellular and chemistry parameters in CSF, conventional culture for bacteria and PCR with sequencing for viral $[3,17]$ studies on the VOCs in the CSF have not been reported. Thus, our study used a gas chromatography-mass spectrometry (GC-MS) method combined with multivariate data analysis to discriminate the VOCs from bacterial and viral ME samples and to discover the potential biomarkers for bacterial and viral ME in CSF VOCs.

\section{Methods}

\section{Human Subjects}

This experiment is consistent with the Declaration of Helsinki [18]. This study's protocol was approved by the Ethics Committee at the First Affiliated Hospital of Harbin Medical University (No.201314). Every patient who participated in the experiment signed written informed consent and agreed with participating in this experiment before entering the experiment. This study was conducted over a period of approximately 11 months (December 2013-October 2014) at the Department of Anesthesiology and Neurology in the First Affiliated Hospital of Harbin Medical University.

This study involved a total of 58 patients with ME, including 16 patients in the bacterial ME group and 42 patients in the viral ME group. The demographic characteristics are summarized in Table 1.

All selected subjects met the entry criteria and exclusion criteria strictly established in this study. The following inclusion criteria were utilized for these individuals: (1) signing informed consent and consenting to participate in the experiment; (2) age from 18 to 70 years; and (3) culture of pathogens in the CSF was diagnosed as bacterial ME or viral ME. Exclusion criteria were as follows: (1) pregnant, breast-feeding, or unable to exclude women with possible pregnancies; (2) identification of known congenital diseases; (3) family history of mental illness; (4) chronic inflammatory diseases; (5) any manifestations of acute illness in the last 2 weeks; (6) history of infectious diseases; (7) patients whose CSF samples cannot be collected; (8) patients with benign or malignant tumors; (9) those receiving radiation and chemotherapy; and (10) those taking special drugs. The experimental implementation of this experiment is similar to the experimental method of a previously published clinical trial article[14].

\section{CSF sample collection}


A CSF specimen collected by lumbar puncture was taken with a 10-ml centrifuge tube, and $2 \mathrm{ml}$ of CSF was transferred to a silylated SPME vacuum bottle. The test was completed within 1 hour after the specimen was collected.

\section{Solid-Phase Microextraction (SPME)}

A manual SPME holder with 75-um-thick carboxyl/polydimethylsiloxane (CAR/PDMS) fibers was purchased from Supelco (Bellefonte, USA). The SPME fiber was inserted into a vial and exposed to a gas sample at $40^{\circ} \mathrm{C}$ for 20 minutes. Subsequently, the SPME holder was placed in the gas chromatograph inlet for desorption.

\section{GC/MS Analysis}

The GC/MS (Shimadzu GC-MS QP 2010, Shimadzu, Japan) used in this study was a DB-5MS (length 30 $m$ * ID 0.250 * film thickness 0.25 um; Agilent Technologies, USA) plot column. Injections were performed in splitless mode, with a $200^{\circ} \mathrm{C}$ injector temperature. The carrier gas was helium, with a purity of $99.999 \%$ and a constant flow rate of $2 \mathrm{ml} \mathrm{min}^{-1}$. The column temperature was held at $40^{\circ} \mathrm{C}$ for $1 \mathrm{~min}$ to concentrate the hydrocarbons at the head of the column and increased by $5^{\circ} \mathrm{C} \min ^{-1}$ to $200^{\circ} \mathrm{C}$ for $1 \mathrm{~min}$, and then the temperature of the column was increased to $230^{\circ} \mathrm{C}$ for 3 minutes at a temperature increase rate of $15^{\circ} \mathrm{C}$ per minute. The MS was performed in full-scan mode, using a scan range from 35-350 amu. The ion source was maintained at $230^{\circ} \mathrm{C}$, and an ionization energy of $70 \mathrm{eV}$ was used for each measurement.

\section{Extraction and Pretreatment of the GC/MS Raw Data}

The original data were converted into the CDF format (NetCDF) using Shimadzu GCMS Postrun Analysis software. Subsequently, the data were processed using the XCMS toolbox. The default settings for XCMS parameters were as follows: $x c m s S e t ~(f w h m=8$, snthresh $=6$, $\max =200$ ); retcor (method ="linear," family ="gaussian,"plottype ="mdevden"). The retention index of each peak was calculated from the ratio of the exact retention time of each peak and the existing retention time of the corresponding series of alkanes C4-C40. Afterwards, the corresponding mass spectrum of all the compounds detected and their corresponding accurate retention time index were compared with the mass spectrogram and the retention time index in the National Institute of Standards and Technology (NIST) Library 2.0 (2011) to perform peak identification.

\section{Statistical Analysis}

Prior to statistical analysis, we performed total area normalization for each sample. Then, the normalized data were analyzed using the SIMCA-P 11.5 platform for multivariate data analysis and modeled. Principal component analysis (PCA) and orthogonal partial least-squares discriminant analysis (OPLSDA) were performed to observe the grouping trend and the outliers of the data. Avoiding the occurrence of overfitting, the permutation tests with 100 iterations were performed to validate the 
supervised model. In addition, for the purpose of determining the significance of each metabolite, the nonparametric Kruskal-Wallis rank sum test was performed. On account of the variable importance in the projection (VIP) values calculated from the OPLSDA model and the P-values from the nonparametric test, potential metabolic biomarkers were obtained using the thresholds of 1.2 and 0.05 , respectively.

\section{Results}

\section{Bacterial ME Patients versus Viral ME Patients.}

GC/MS was utilized to analyze the metabolites in the CSF from 16 patients in the bacterial ME group and 42 patients in the viral ME group. Based on the ion peaks in the resulting chromatogram, a total of 361 variables were detected. In the two-dimensional PCA score plot (8 principal components, R2X $=0.834$, $\mathrm{Q} 2=0.632$ ), there was no clear separation trend between the two groups of data (Figure 1). The OPLSDA score plot ( 2 components, $\mathrm{R} 2 \mathrm{X}=0.503, \mathrm{R} 2 \mathrm{Y}=0.523, \mathrm{Q} 2=0.387$ ) demonstrated a separation between the bacterial ME group patients and the viral ME group patients using one predictive component and three orthogonal components (Figure 2). Additionally, as calculated from the permutated data, the R2 and Q2 values were lower than the original values in the validation plot, which confirmed the validity of the supervised model (Figure 3).

\section{Potential Biomarkers}

The potential biomarkers in both the bacterial ME group and the viral ME group were determined by the VIP value calculated from the OPLSDA model and the P-values from the nonparametric test. Among the significant metabolites identified using the VIP values and the $P$ values, 2 differential metabolites are annotated using the NIST 11 database with a similarity threshold of $75 \%$. Two significant substances were found in the bacterial ME group and the viral ME group ( $P<0.05$, Table 2, Figure 4): ethylene oxide and phenol. Ethylene oxide was significantly greater in the group of bacterial ME patients than in the viral ME group $(P<0.05)$. Moreover, significantly increased levels of phenol were detected in the group of viral ME patients than in the bacterial ME group $(P<0.05)$.

\section{Discussion}

CNS infectious diseases have a high morbidity and mortality [1]. The incidence of bacterial ME and viral ME accounts for the majority of CNS infectious diseases [2,3]. However, typical pathogen identification methods have disadvantages of being time-consuming and expensive and have low sensitivity and poor specificity ${ }^{[7,8]}$; therefore, distinguishing bacterial ME and viral ME has been a clinical problem for a long time [3].

As a developing novel tool for the non-invasive detection of various disease states in recent years, VOC analysis has been applied in different body fluids, such as blood, urine, and saliva [12-15]. VOC analysis is important in the diagnosis of respiratory infections as an accurate and rapid method and has clinical value in guiding the use of antibiotics [19-21]. However, there are no studies to discuss the VOCs in CSF. 
Considering that the CSF is a type of human body fluid and is also a type of bacterial and viral microbiological culture in vivo, our study discriminated the VOCs of bacterial and viral ME samples to discover potential biomarkers for bacterial and viral ME in CSF VOCs, which may provide a detection method with greater specificity and accuracy.

Previous studies have determined that significant VOCs released by the pathogens mainly include aldehydes, alcohols, ketones, lipids and acids [22]. In our study, a total of 361 metabolites were detected from the bacterial and viral ME group patients. There was an obvious difference in the ion peaks in the resulting chromatograms among them; however, to serve as a potential biomarker, the VIP value must be greater than 1.2 and the $P$ value less than 0.05 .

Filipiak et al. [25] found that the potential volatile biomarkers produced by E. coli include an aromatic compound - Benzonitrile. E. coli is a pathogen that causes bacterial ME. Raţiu et al. [26] found that the significant VOC of E. coli is indole, because it is generated at three time points and is also the most produced VOC. Other studies have also confirmed this finding [27-29]. Indole is an aromatic heterocyclic organic compound with the formula $\mathrm{C}_{8} \mathrm{H}_{7} \mathrm{~N}$. 5-Hydroxytryptamine (5-HT) is a monoamine neurotransmitter that is mainly decarboxylated by tryptophan in the human body. E. coli produces tryptophan decarboxylase and degrades tryptophan to indole and other compounds [30]. Karami et al. [19] found that 2,3-pentanedione, 1-decene, 1,3-dimer, 2,5-dimethylpyrazine, ethyl butyrate and cyclohexene are specific VOCs of S. aureus in vitro. It is believed that the VOCs released by S. aureus have the ability to degrade amino acids in its growth environment [27]. A study conducted by lleanaAndreea et al. [10] described the significant VOCs of Mycobacterium tuberculosis, which were naphthalene, 1-methyl, -cyclohexane, heptane, 2,2,4,6,6-pentamethyl benzene, 1,3,5-trimethyl- and 4methyl, but the specific mechanism was not yet clear. In the study by Chen et al [31], the growth of $L$. monocytogenes was found to be related to 3-hydroxy-2-butanone; however, the specific mechanism was not yet clear. The studies of other bacteria causing bacterial ME should be carried out in large quantities.

Studies have shown that the mechanism underlying viral pathogenicity is the induction of antiviral activity in host cells. After the virus invades the body, it can activate the innate immune cells to secrete a large number of pro-inflammatory factors, including interleukin (IL)-1, IL-6, IL-12 and tumor necrosis factor-a [32]. These factors can induce inflammation, which is characterized by The acute phase of inflammation promotes the production of complement proteins, and the final result is the induction of apoptosis [33]. Abd et al. [9] found numerous significant VOCs in bacterial and viral microbiological cultures. Dodecane (a type of alkane) was found in the virus group, which may be related to the degradation of cell membranes by lipid peroxidation and the conversion of polyunsaturated fatty acids to volatile alkanes, but the true source is unknown.

The most common pathogens of bacterial ME are L. coli, S. aureus, and Haemophilus influenzae [3]. The major viral causes of viral ME include enterovirus (EV), cytomegalovirus (CMV), and herpes simplex virus type 1 (HSV-1) [3]. The significant VOCs released by the pathogen provide [9], and every patient has different pathogens. In our study, we analyzed the VOCs of all ME patients but not those of the 
pathogens. The substances in microbiological cultures in vitro and in vivo are not exactly the same. In the future, we will culture the CSF of ME (especially common pathogens) and study the specificity of the significant VOCs released by different pathogens.

There are several limitations in this study. There is no specific division of the patients' age structure, and more accurate research needs further practice. Due to the limitations of the conditions, this study did not analyze the VOCs of healthy volunteers. Last but not least, this study is a prospective pilot study, and there are few studies discussing the VOCs produced by viral cultures; therefore, a large number of studies are required to explore and verify the results of this study.

\section{Conclusions}

Ethylene oxide and phenol may be potential biomarkers to distinguish bacterial ME and viral ME. VOC analysis of CSF may be used as a supporting tool for clinical diagnosis.

\section{Abbreviations}

CNS: Central nervous system; ME: meningitis/encephalitis; CSF: Cerebrospinal fluid; BBB: blood-brain barrier; BCSFB: blood-cerebrospinal fluid barrier; VOCs: Volatile organic compounds; GC: gas chromatography; MS: mass spectrometry; E-noses: electronic nose; IMS: ion mobility spectrometry; GCMS: gas chromatography-mass spectrometry; SPME: solid-phase microextraction; NIST: national institute of standards and technology; PCA: principal component analysis; OPLSDA: orthogonal partial leastsquares discriminant analysis; VIP: variable importance in the projection; 5-HT: 5-hydroxytryptamine; IL: interleukin; TNF-a: tumor necrosis factor-alpha; EV: enterovirus; CMV: cytomegalovirus; HSV-1: herpes simplex virus type 1 .

\section{Declarations}

\section{Authors' contributions}

LG and ZZQ were major contributors in writing the manuscript, YW and KLY analyzed the patient data. $\mathrm{XYZ}$, YHL, ML, GYW, NNG and MYY collected patient data, EYL and CSW put forward ideas. All authors read and approved the final manuscript.

\section{Acknowledgements}

This work was supported by Nn10 program and Distinguished Young Scholars Fund of Harbin Medical University Cancer Hospital; the Yuweihan Fund for Distinguished Young Scholars of Harbin Medical University; Harbin Science and Technology Innovation Scholars Fund (2017RAXXJ087); Heilongjiang Province Postdoctoral Research Fund. 


\section{Competing interests}

The authors declare no competing financial interests.

\section{Availability of data and materials}

All data are included in the manuscript. However, the raw data used and/or analyzed in the presen study are available from the corresponding author on reasonable request.

\section{Ethics approval and consent to participate}

This experiment is consistent with the Declaration of Helsinki. This study's protocol was approved by the Ethics Committee at the First Affiliated Hospital of Harbin Medical University (No.201314). Every patient who participated in the experiment signed informed consent before entering the experiment.

\section{Consent for publication}

Not applicable.

\section{Funding}

This work was supported by Nn10 program and Distinguished Young Scholars Fund of Harbin Medical University Cancer Hospital; the Yuweihan Fund for Distinguished Young Scholars of Harbin Medical University; Harbin Science and Technology Innovation Scholars Fund (2017RAXXJ087); Heilongjiang Province Postdoctoral Research Fund. The design of the study and material supplements were supported by Nn10 program and Distinguished Young Scholars Fund of Harbin Medical University Cancer Hospital, and the interpretation of data and writing editing service of this manuscript were supported by a grant of the Yuweihan Fund for Distinguished Young Scholars of Harbin Medical University; Harbin Science and Technology Innovation Scholars Fund (2017RAXXJ087); Heilongjiang Province Postdoctoral Research Fund.

\section{References}

1. Rozell JM, Mtui E, Pan YN, Li S. Infectious and inflammatory diseases of the central nervous systemthe spectrum of imaging findings and differential diagnosis. Emerg Radiol. 2017;24(6):619-33. 
2. Lozano R, Naghavi M, Foreman K, Lim S, Shibuya K, Aboyans V, Abraham J, Adair T, Aggarwal R, Ahn SY,et al. Global and regional mortality from 235 causes of death for 20 age groups in 1990 and 2010: a systematic analysis for the Global Burden of Disease Study 2010. Lancet. 2012;380(9859):2095-128.

3. Leber AL, Everhart K, Balada-Llasat JM, Cullison J, Daly J, Holt S, Lephart P, Salimnia H, Schreckenberger PC, DesJarlais S,et al. Multicenter Evaluation of BioFire FilmArray Meningitis/Encephalitis Panel for Detection of Bacteria, Viruses, and Yeast in Cerebrospinal Fluid Specimens. J Clin Microbiol. 2016;54(9):2251-61.

4. Glimåker $M$, Johansson B, Grindborg Ö, Bottai M, Lindquist L, Sjölin J. Adult bacterial meningitis: earlier treatment and improved outcome following guideline revision promoting prompt lumbar puncture. Clin Infect Dis. 2015;60(8):1162-9.

5. Hasbun R, Rosenthal N, Balada-Llasat JM, Chung J, Duff S, Bozzette S, Zimmer L, Ginocchio CC. Epidemiology of Meningitis and Encephalitis in the United States, 2011-2014. Clin Infect Dis. 2017;65(3):359-63.

6. Hakan Erdem, Yasemin Cag, Derya Ozturk-Engin, Sylviane Defres, Selcuk Kaya, Lykke Larsen, Mario Poljak, Bruno Barsic, Xavier Argemi, Signe Maj Sørensen, Anne Lisbeth Bohr, Pierre Tattevin,et al. Results of a multinational study suggest the need for rapid diagnosis and early antiviral treatment at the onset of herpetic meningoencephalitis. Antimicrob Agents Chemother. 2015;59(6):3084-9.

7. McGill F, Griffiths MJ, Solomon T. Viral meningitis: current issues in diagnosis and treatment. Curr Opin Infect Dis. 2017;30(2):248-56.

8. Spanos A, Harrell FE Jr, Durack DT. Differential diagnosis of acute meningitis. An analysis of the predictive value of initial observations. JAMA. 1989;262(19):2700-7.

9. Abd El Qader A, Lieberman D, Shemer Avni Y, Svobodin N, Lazarovitch T, Sagi O, Zeiri Y. Volatile organic compounds generated by cultures of bacteria and viruses associated with respiratory infections. Biomed Chromatogr. 2015; 29: 1783-90.

10. Ileana-Andreea Ratiu, Tomasz Ligor1, Victor BocosBintintan, Bogusław Buszewski. Mass spectrometric techniques for the analysis of volatile organic compounds emitted from bacteria. 2017; 9(14): 1069-92.

11. Nakhleh MK, Amal H, Jeries R, Broza YY, Aboud M, Gharra A, Ivgi H, Khatib S, Badarneh S, Har-Shai L,et al. Diagnosis and Classification of 17 Diseases from 1404 Subjects via Pattern Analysis of Exhaled Molecules. ACS Nano. 2017 Jan 24; 11(1): 112-125.

12. Ramesh P. Arasaradnam, Eric Westenbrink, Michael J. McFarlane, Ruth Harbord, Samantha Chambers, Nicola O’Connell, Catherine Bailey, Chuka U. Nwokolo, Karna D. Bardhan, Richard Savage,et al. Differentiating Coeliac Disease from Irritable Bowel Syndrome by Urinary Volatile Organic Compound Analysis-A Pilot Study. PLoS One. 2014; 9(10):e107312.

13. Arasaradnam RP, McFarlane MJ, Ryan-Fisher C, Westenbrink E, Hodges P, Thomas MG, Chambers S, O'Connell N, Bailey C, Harmston C,et al. Detection of colorectal cancer (CRC) by urinary volatile organic compound analysis. PLoS One. 2014; 9(9): e108750. 
14. Wang D, Wang C, Pi X, Guo L, Wang Y, Li M, Feng Y, Lin Z, Hou W, Li E. Urinary volatile organic compounds as potential biomarkers for renal cell carcinoma. Biomed Rep. 2016; 5(1): 68-72.

15. Wang M, Xie R, Jia X, Liu R. Urinary Volatile Organic Compounds as Potential Biomarkers in Idiopathic Membranous Nephropathy. Med Princ Pract. 2017; 26(4): 375-380.

16. Goeminne PC, Vandendriessche T, Van Eldere J, Nicolai BM, Hertog ML, Dupont LJ. Detection of Pseudomonas aeruginosa in sputum headspace through volatile organic compound analysis. Respir Res. 2012; 13: 87.

17. Dąbrowski P, Jurkiewicz J, Czernicki Z, Koszewski W, Jasielski P. Polymerase chain reaction based detection of bacterial 16S rRNA gene in the cerebrospinal fluid in the diagnosis of bacterial central nervous system infection in the course of external cerebrospinal fluid drainage. Comparison with standard diagnostics currently used in clinical practice. Neurol Neurochir Pol. 2017;51(5):388-394.

18. Gandevia, B. Tovell, A. Declaration of Helsinki. Med J Aust. 1964;2: 320-321.

19. Karami N, Mirzajani F, Rezadoost H, Karimi A, Fallah F, Ghassempour A, Aliahmadi A. Initial study of three different pathogenic microorganisms by gas chromatography-mass spectrometry. Version 3. F1000Res. 2017; 6: 1415.

20. Boots AW, Smolinska A, van Berkel JJ, Fijten RR, Stobberingh EE, Boumans ML, Moonen EJ, Wouters EF, Dallinga JW, Van Schooten FJ. Identification of microorganisms based on headspace analysis of volatile organic compounds by gas chromatography-mass spectrometry. J Breath Res. 2014; 8(2): 027106.

21. Karami N, Karimi A, Aliahmadi A, Mirzajan F, Rezadoost H, Ghassempour A, Fallah F. Identification of bacteria using volatile organic compounds. Cell Mol Biol (Noisy-le-grand). 2017; 63(2): 112-121.

22. Bos LD, Sterk PJ, Schultz MJ. Volatile metabolites of pathogens: a systematic review. PLoS Pathog. 2013;9(5):e1003311.

23. Filipiak W, Sponring A, Baur MM, Ager C, Filipiak A, Wiesenhofer H, Nagl M, Troppmair J, Amann A. Characterization of volatile metabolites taken up by or released from Streptococcus pneumoniae and Haemophilus influenzae by using GC-MS. Microbiology. 2012;158(Pt 12):3044-53.

24. Wojciech Filipiak, Andreas Sponring, Maria Magdalena Baur, Anna Filipiak, Clemens Ager, Helmut Wiesenhofer, Markus Nag, Jakob Troppmair, Anton Aman. Molecular analysis of volatile metabolites released specifically by staphylococcus aureus and pseudomonas aeruginosa. BMC Microbiol. 2012; 12: 113.

25. Filipiak W, Beer R, Sponring A, Filipiak A, Ager C, Schiefecker A, Lanthaler S, Helbok R, Nagl M, Troppmair J,et al. Breath analysis for in vivo detection of pathogens related to ventilator-associated pneumonia in intensive care patients: a prospective pilot study. Journal of breath research. 2015, 9(1): 016004.

26. Raţiu IA, Bocoş-Binţinţan V, Turner M. Discrimination of chemical profiles of some bacterial species by analyzing culture headspace air samples using TD-GC/MS. Cur. Anal. Chem. 2014; 10(4), 488-97.

27. Zhu J, Bean HD, Kuo YM, Hill JE. Fast detection of volatile organic compounds from bacterial cultures by secondary electrospray ionization-mass spectrometry. J Clin Microbiol. 2010; 48(12): 
4426-31.

28. Tait E, Perry JD, Stanforth SP, Dean JR. Identification of volatile organic compounds produced by bacteria using HS-SPME-GC-MS. J Chromatogr Sci. 2014; 52(4): 363-73.

29. Garner CE, Smith S, de Lacy Costello B, White P, Spencer R, Probert CS, Ratcliffe NM. Volatile organic compounds from feces and their potential for diagnosis of gastrointestinal disease. FASEB J. 2007; 21(8): 1675-88.

30. Martino PD, Fursy R, Bret L, Sundararaju B, Phillips RS. Indole can act as an extracellular signal to regulate biofilm formation of Escherichia coli and other indole-producing bacteria. Can $\mathrm{J}$ Microbiol. 2003; 49(7): 443-9.

31. Chen J, Tang J, Shi H, Tang C, Zhang R. Characteristics of volatile organic compounds produced from five pathogenic bacteria by headspace-solid phase micro-extraction/gas chromatography-mass spectrometry. J Basic Microbiol. 2017; 57(3): 228-237.

32. Dahm T, Rudolph H, Schwerk C, Schroten H, Tenenbaum T. Neuroinvasion and Inflammation in Viral Central Nervous System Infections. Mediators Inflamm. 2016;2016:8562805.

33. Heath RB. The pathogenesis of respiratory viral infection. Postgraduate Medicine Journal 1979; 55(640): 122-127.

\section{Tables}

Table-1. Demographic characteristics of the study subjects

\begin{tabular}{lcc}
\hline & Bacterial meningitis/encephalitis group & Viral meningitis/encephalitis \\
& $\square \mathrm{n}=16 \square$ & group \\
& & $\square \mathrm{n}=42 \square$ \\
\hline Age $\llbracket$ mean \pm & $46 \square 17.3 \square$ & $38 \square 14.4 \square$ \\
$\mathrm{SD} \square$ & & 27 \\
male & 8 & 15 \\
female & 8 & 15 \\
\hline
\end{tabular}

Table-2. Related metabolites that exist at abnormal levels in the cerebrospinal fluid between bacterial meningitis/encephalitis patients versus viral meningitis/encephalitis patients.

\begin{tabular}{lllll}
\hline potential biomarkers & RT & VIP & P & FC \\
\hline Ethylene oxide & 1.178328 & 12.8869 & 0.038427 & 0.306375 \\
Phenol & 8.536144 & 1.85754 & 0.041803 & -0.416729 \\
\hline
\end{tabular}


Figures

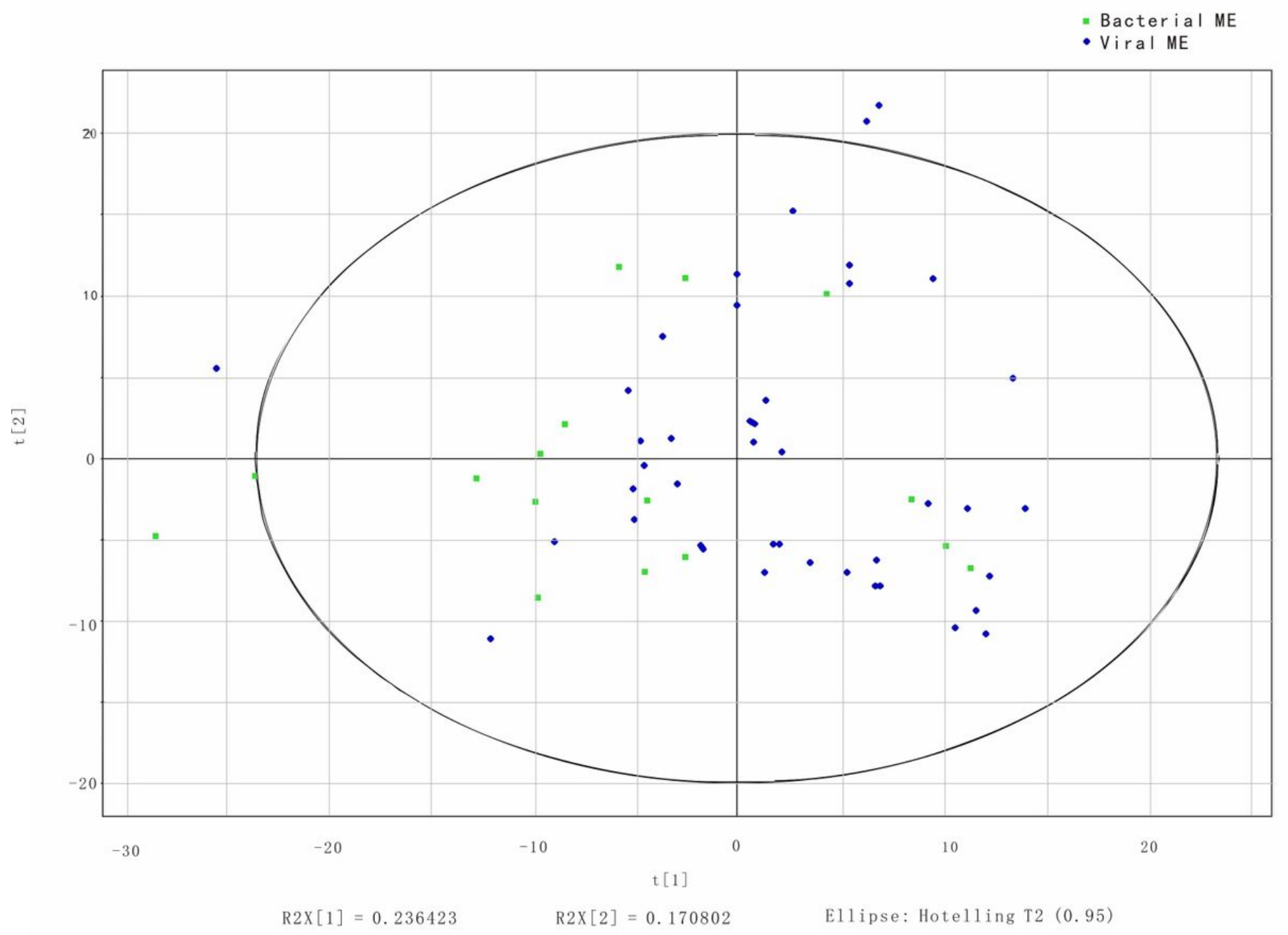

Figure 1

PCA score plot for cerebrospinal fluid samples from bacterial meningitis/encephalitis versus viral meningitis/encephalitis patients (8 components, R2X=0.834, Q2=0.632). 


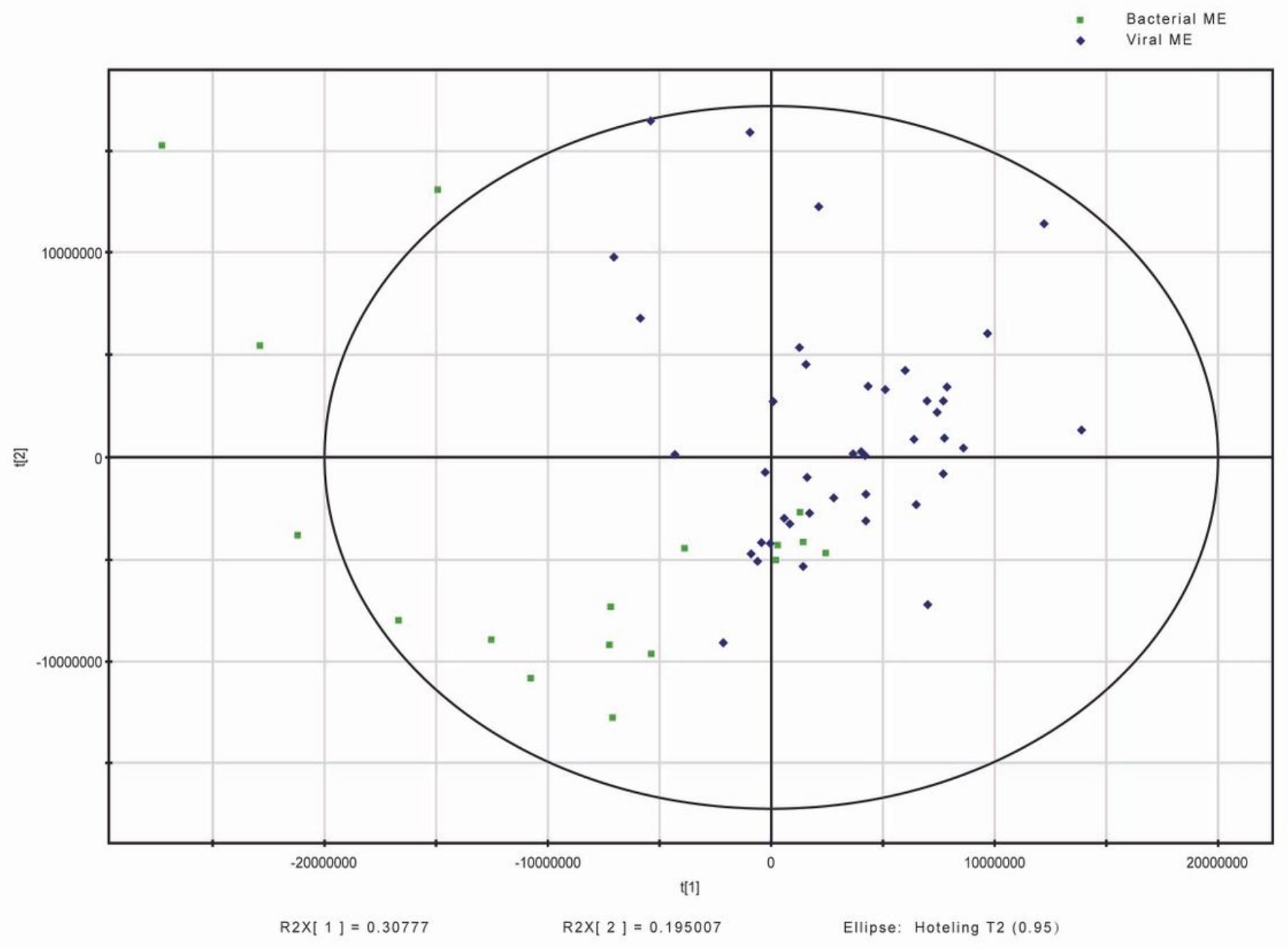

Figure 2

OPLSDA score plot for cerebrospinal fluid samples from bacterial meningitis/encephalitis versus viral meningitis/encephalitis patients ( 2 components, R2X=0.503, R2Y=0.523, Q2=0.387). 


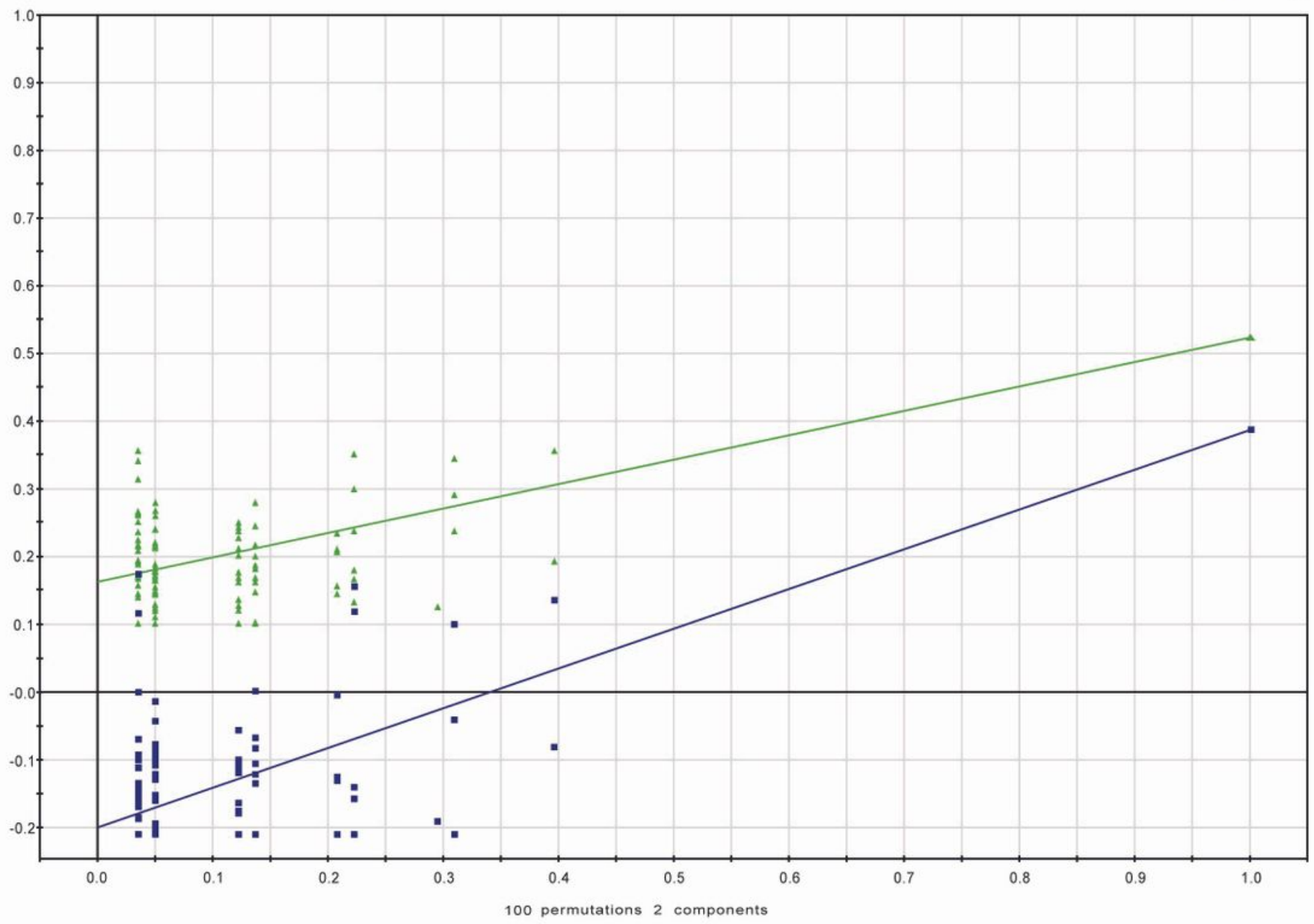

Figure 3

OPLSDA validation plot intercepts for cerebrospinal fluid samples from bacterial meningitis/encephalitis versus viral meningitis/encephalitis patients $₫ \mathrm{R} 2=(0.0,0.162) ; \mathrm{Q} 2=(0.0,-0.2)$. 


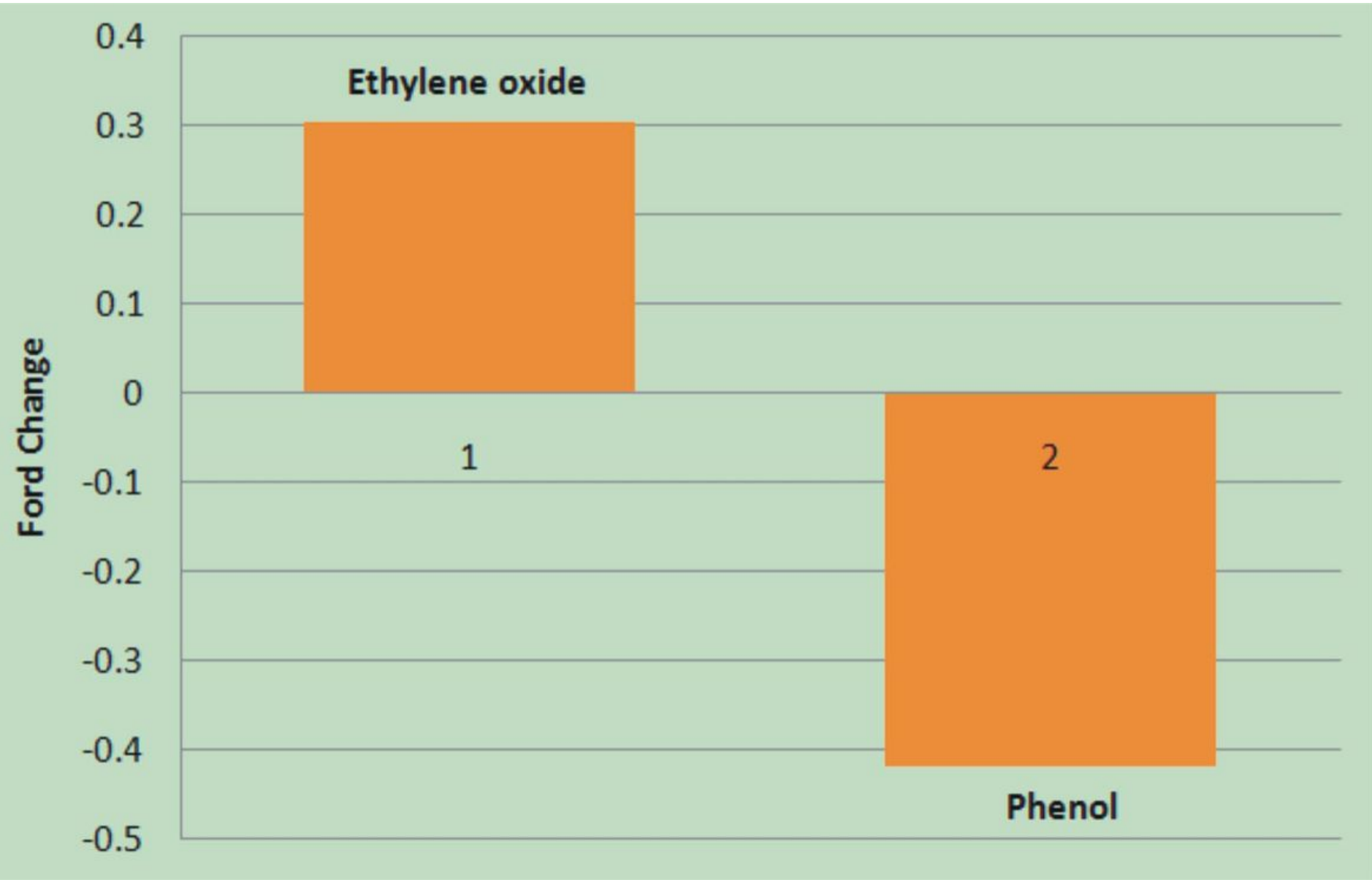

\section{Figure 4}

Related meningitis/encephalitistabolites that exist at abnormal levels in the cerebrospinal fluid between bacterial meningitis/encephalitis patients versus viral meningitis/encephalitis patients. 